\title{
Development of Practical Wrist Rehabilitation Robot by Mirror Effect
}

\author{
Ikuo Yamamoto ${ }^{1, a^{*}}$, Miki Matsui ${ }^{1}$, Naohiro Inagawa ${ }^{2}$ and Takunori Tsuji ${ }^{3}$ \\ Kenji Hachisuka ${ }^{4}$, Futoshi Wada ${ }^{4}$ and Akiko Hachisuka ${ }^{4}$
}

${ }^{1}$ Dept. of Mechanical Science, Nagasaki Univ., Graduate School, 1-14 Bunkyomachi, Nagasaki-shi, Nagasaki 852-8521, Japan

${ }^{2}$ Dept. of Media Technology, Nippon Bunri Univ., 1727 Ichigi, Oita-shi, Oita 870-0397, Japan

${ }^{3}$ Logical Product Corporation, 2-25-5 Matoba, Minami-ku, Fukuoka-shi, Fukuoka 811-1314, Japan

${ }^{4}$ Dept. of Rehabilitation Medicine, Univ. of Occupational and Environmental Health, 1-1 Iseigaoka, Yahatanishi-ku, Kitakyushu-shi, Fukuoka 807-8555, Japan

aiyamamoto@nagasaki-u.ac.jp

Keywords: Rehabilitation robot, Mirror effect, Wrist training

Abstract. The authors have developed wrist rehabilitation robot for hemiplegic patients. The robot is much effective for repetitive rehabilitation, and useful for patients. Mirror effect control system is developed based on the experience that a patient can easily move a limb while moving a limb on the other side. The effectiveness is successfully confirmed by the practical test in the hospital.

\section{Introduction}

The effective wrist rehabilitation robot shown in Fig.1 has been developed at practical level for hemiplegic patients, who need repetitive rehabilitation training to improve the function of upper and lower extremities and to increase the range of motion. The effectiveness of repetitive rehabilitation training is shown in medical researches [1-8]. The mild physically disabled patients can undergo self-training with such rehabilitation tools as blocks. In contrast to it, the massive physically disabled patients generally undergo forced training with training support devices. However, forced training fails to be higher effective because patients cannot exercise on their own active mind. Therefore, the effect of the treatment is unable to surpass the critical limit. Although occupational therapists can give the patients undivided attention, the number of occupational therapists is limited and there is a compelling need to reduce their work load. Many research results for rehabilitative training robot have been reported [9-14], however, it is often the case that the robot system is large and the process is complicated. Therefore, it is difficult for patients to feel unrestrained to use the robot system. Then, the research is aimed at developing a rehabilitative robot that enables the effective training of patient. The training robot conducts several modes of training. Specially, the paretic hand can undergo the rehabilitative training by the other non-paretic hand based on mirror effect control system.

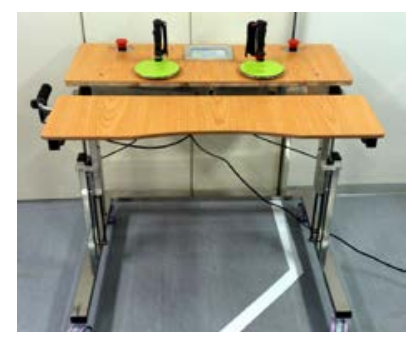

Fig.1 The effective wrist rehabilitation robot

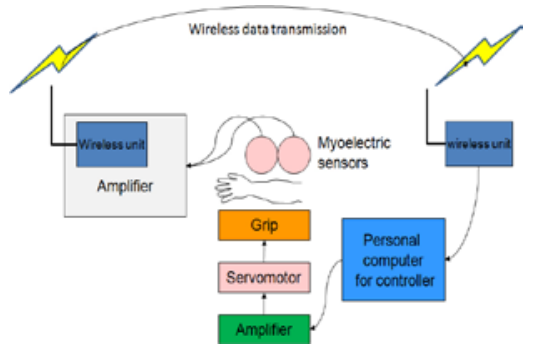

Fig. 2 The rehabilitative training robot using myoelectric sensors 


\section{System configuration}

The developed rehabilitative robot consists of passive, constant torque and autonomous training modes. In addition to that, the authors have developed the training robot that has a function to detect the myoelectric potential by myoelectric sensors on patient's muscle, and analyze the patient's intention to flex their wrist shown in Fig.2, Fig3 and Fig4. The training robot can provoke training through the function, so that patients can undergo effective training shown in Fig.5 [15-17]. The rehabilitative training robot consists of grip, actuator, biological signal detector, biological signal processor, training controller and both-wrist system. Grip is held by patient during training. The training movement is generated by servomotor as the actuator.

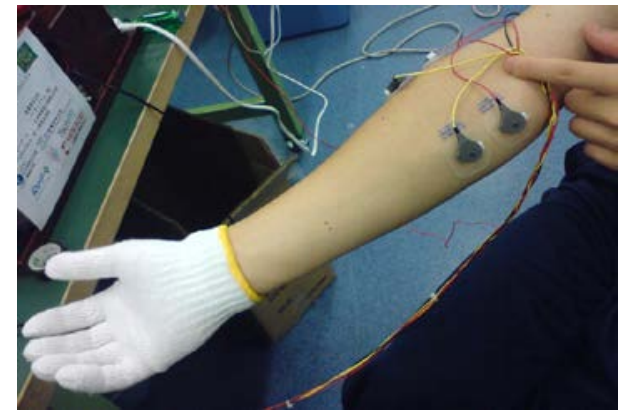

Fig.3 The myoelectric sensors

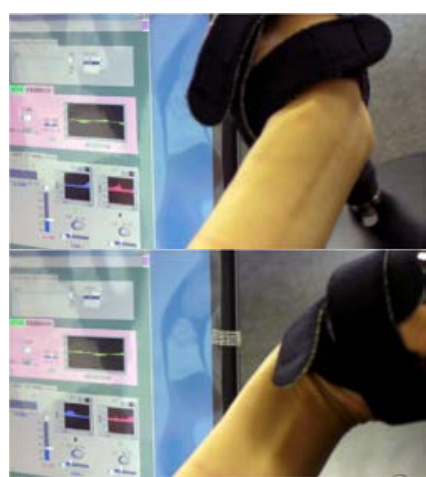

Fig.5 The developed rehabilitation robot in an excited condition

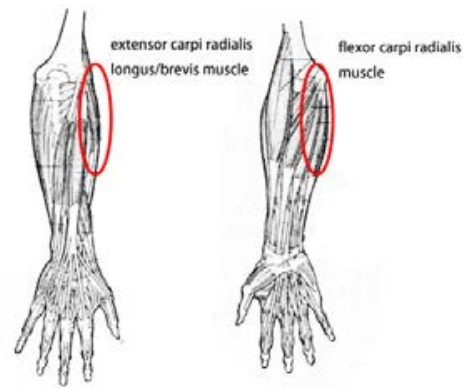

Fig.4 The extensor carpi radialis longus/brevis muscle and flexor carpi radialis muscle

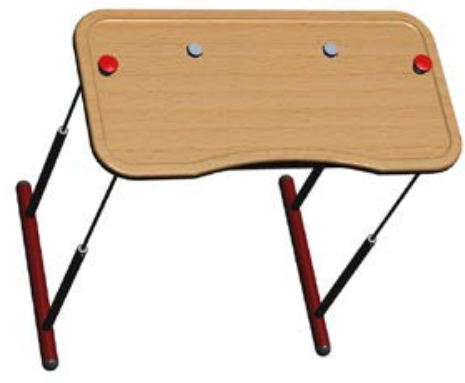

Fig.6 The CAD design of the new rehabilitative training robot for hospital use

\section{System development}

The system is firstly designed by CAD shown in Fig.6. Through adjustments of manufacturing for safety use, the wrist rehabilitation robot has been developed shown in Fig.1. The picture of grip and computer display is shown in Fig.7. The robot is small desk size and is easy to carry. Therefore, the training can be performed in a small space in the hospital and at home. The passive mode is the mode of palmar and dorsal flexion motions started by the start button shown in Fig.7. The constant torque mode is the mode that patients operate the grip under the constant torque. The autonomous training mode has two main functions. One function is that the patients operate an arbitrary grip A of one hand in Fig.8. The palmar and dorsal flexion motions of the other hand's grip B and grip A itself are started autonomously. The other function is that the patients operate an arbitrary grip to palmar flexion direction A the palmar flexion motion B is started in Fig.9, and that the dorsal flexion motion is started when they operate it to dorsal flexion direction. 


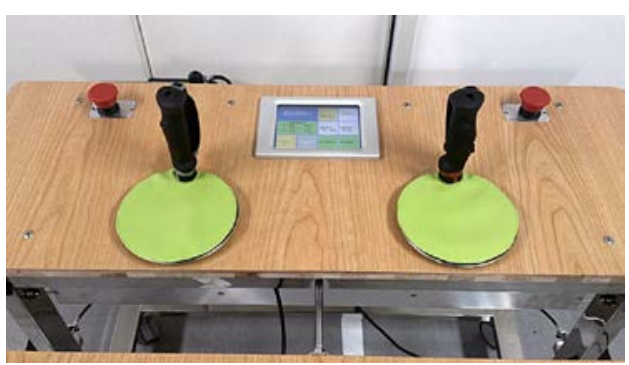

Fig.7 The grip and computer display

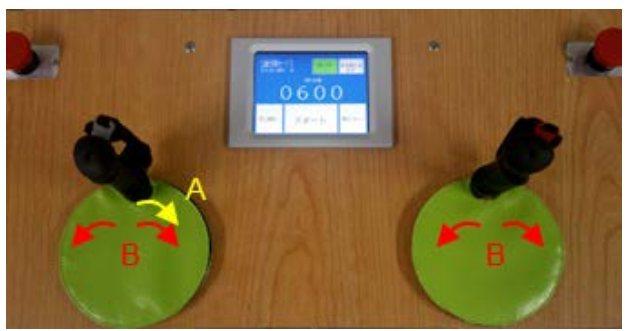

Fig.8 The autonomous training mode 1

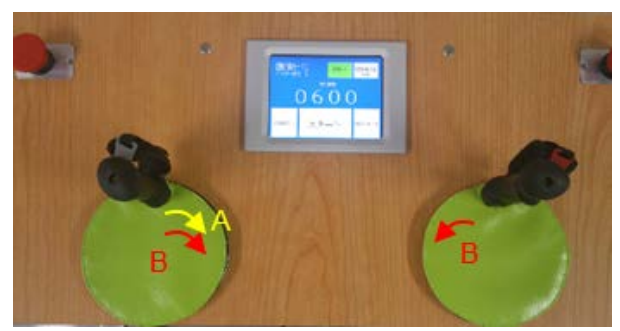

Fig.9 The autonomous training mode 2

\section{System test}

The rehabilitation robot system has been tested in the hospital. The user-friendly screen for patients with easily recognizable touch panels, therefore, it can avoid operational error when used by patients on their own. The training data can be stock for each patient, and re-start training is possible when patient stopped training. Also, the height of desk can be adjusted smoothly manually according to the height of patients. The limit switch of maximum flexion angle of patients and emergent switch to stop the servomotors are equipped with the system for safety. The most effective function is mirror effect training. It is found that mirror effect motion is effective in rehabilitation of patients. The control system is equipped under each circular grip of hands and mirror effect control motion adjustable to patient's state is conducted, therefore, the paretic hand can learn how to move by the real time teaching data of non-paretic hand. The same time interval of flexion training of both wrists at the different speed of rotation can be realized by the control system.

\section{Conclusion}

The practical wrist rehabilitation robot has been developed and is used in the hospital. The robots are introduced and applied to several hospitals, and the authors get clinical data to complete the research. Also, the authors improve more functions according to the requirement from medical doctor, therapists and patients.

\section{Acknowledgement}

A part of study was supported by Grant-in-Aid for Scientific Research (B) and FAIS research. The authors would like to express their sincere gratitude to Arizono Co., Etou Co., and related personnel in the hospital and university for the research and development.

\section{References}

[1] S. Saeki, Y. Matsushima, K. Hachisuka, "Cortical activation during robotic therapy for a severely affected arm in a chronic stroke patient: a case report,” J. UOEH, vol. 30, no. 2, pp. 159-165, 2008. 
[2] G.B. Prange, M.J.A. Jannink, C.G.M. Groothuis-Oudshoon, H.J. Hermens, M.J. IJzerman, "Systematic review of the effect of robot-aided therapy on recovery of the hemiparetic arm after stroke,” J. Rehabil Res Dev, vol. 43, pp. 171-184, 2006.

[3] H.I. Krebs, N. Hogan, B.T. Volpe, M.L. Aisen, L. Edelstein, C. Diels, “Overview of clinical trials with MIT-MANUS: A robot-aided neuro-rehabilitation facility,” Technol. Health Care, vol. 7, pp. 419-423, 1999.

[4] C.G. Burgur, P.S. Lum, P.C. Shor, H.F. Machiel Van der Loos, "Development of robots for rehabilitation therapy: The Palo Alto VA/Standard experience,” J. Rehabil. Res. Dev., vol. 37, pp. 376-388, 2000.

[5] S. Masiero, A. Celia, G. Rosati, M. Armani, "Robotic-assisted rehabilitation of the upper limb after acute stroke,” Arch. Phys. Med. Rehabil., vol. 88, pp. 142-149, 2007.

[6] S. Hesse, G. Schulte-Tiggers, M. Konrad, M.A. Bardeleben, C. Werner, "Robot-assisted arm trainer for the passive and active practice of bilateral forearm and wrist movements in hemiparetic subjects,” Arch. Phys. Med. Rehabil., vol. 84, pp. 915-920, 2003.

[7] S. Hesse, C. Werner, M. Pohl, S. Rueckriem, J. Mehrholz, M.L. Lingnau, “Computerized arm training improves the motor control of the severely affected arm after stroke: a single-blinded randomized trial in two centers," Stroke, vol. 36, pp. 1960-1966, 2005.

[8] M. Ferraro, J.J. Palazzolo, J. Krol, H.I. Krebs, N. Hogan, B.T. Volpe, "Robot-aided sensorimotor arm training improves outcome in patients with chronic stroke,” Neurology, vol. 61, pp. 1604-1607, 2003.

[9] Guan De Lee, Wei-Wen Wang, Kai-Wen Lee, Sheng-Yen Lin, Li-Chen Fu, Jin-Shin S. Lai, Wen-Shiang Chen, Jer-Junn J. Luh, "Arm exoskeleton rehabilitation robot with assistive system for patient after stroke," Proc. of ICCAS, pp. 1943-1948, 2012.

[10] Hong-Bo B. Wang, Ning Du, Huina Hou, Jiangshun Zhao, Hongnain N. Yu, "An upper limb rehabilitation robot based on 3-RRC parallel mechanism," Proc. of ICAMechS, pp. 668-673, 2012.

[11] Xikai Tu, Jian Huang, Liguo Yu, Qi Xu, Jiping He, "Design of a wearable rehabilitation robot integrated with functional electrical stimulation," Proc. of IEEE RAS \& EMBS BioRob, pp. 1555-1560, 2012.

[12] Lei Shi, Kun Jiang, Qiang Wang, Zhen Liu, "A New Method of Quantitative Rehabilitation Rating in Robot-Assisted Recovery System," Proc. of ICINIS, pp. 278-281, 2012.

[13] Yongsuk Kang, Doyoung Jeon, "Rehabilitation robot control using the VSD method," Proc. of SII, pp. 192-197, 2012.

[14] Chao Lv, Le Xie, Wei Shao, Hai-long Yu, Yuan Wang, Jin-wu Wang, Ning Nan, "Development of a Rehabilitation Robot for Upper-Limb Movements," Intelligent Robotics and Applications, Lecture Notes in Computer Science, Vol.7507, pp. 96-102, 2012.

[15] I.Yamamoto, N.Inagawa, K.Hachisuka, et al., "Development of compact rehabilitation robot for a wrist using biological signal”, Proc.of IEEE CME, pp.557-560, 2012.

[16] I.Yamamoto, N.Inagawa, K.Hachisuka, F.Wada, A.Hachisuka, "Development of a Rehabilitative Training Robot for Both Wrists”, Proc.of 2013 ICME, pp.280-284, 2013.

[17] I.Yamamoto, N.Inagawa, M.Matsui, K.Hachisuka, F.Wada, A.Hachisuka, "Research and Development of Compact Wrist Rehabilitation Robot System”, Bio-Medical Materials and Engineering 24, pp.123-128, 2014. 